\title{
ARTÍCULOS
}

\section{Proceso de enseñanza aprendizaje de la lengua inglesa en escuelas públicas chilenas: ¿Producción o reproducción?}

\author{
The learning teaching process of English as a foreign language \\ in Chilean Public Schools: Production or reproduction?
}

\author{
Yilorm, Yasna \\ Instituto de Lingüística y Literatura \\ Facultad de Filosofía y Humanidades \\ Universidad Austral de Chile \\ yyilorm@uach.cl \\ Tel.: (56) 63-2221425.
}

\begin{abstract}
RESUMEN
Las bases curriculares para la enseñanza de la lengua inglesa en Chile no siempre concuerdan con el proceso de enseñanza aprendizaje en el aula. El Ministerio de Educación propone el desarrollo de la competencia comunicativa y contrariamente, según la evidencia científica, las clases de inglés en el sector público priorizan la repetición y traducción del léxico e utilización explícita de las estructuras gramaticales. A esto se suma que los componentes humanos y culturales del proceso en estudio no están dialécticamente relacionados entre sí para lograr un fin común. Mediante la revisión de los planes y programas de sexto básico, la autora analiza críticamente estas disonancias y declara la urgente necesidad de definir, de manera cooperativa, los fundamentos teóricos del proceso de enseñanza aprendizaje de esta lengua extranjera en Chile, con el fin de alcanzar la armonía de dichos componentes y permitir, en consecuencia, una comunicación natural y eficaz.
\end{abstract}

Palabras claves: Inglés, comunicación en el aula, educación tradicional, disonancia, fundamentos teóricos

\begin{abstract}
The national curriculum for teaching English in Chile does not always correspond to the learning teaching process in the classroom. The Ministry of Education proposes the development of students' communicative competence, and contrarily, according to scientific evidence, English lessons in public schools are focused on lexicon repetition and translation and on the explicit use of grammar structures. In addition, the human and cultural components of the learning teaching process are not dialectically related in order to reach a common goal. Through the analysis of the sixth grade curriculum, the author critically analyses these contradictions and states the urgent need to define, in a cooperative manner, the theoretical fundaments of the teaching learning process of English as a foreign language in Chile, with the purpose of reaching components harmony and therefore, allow a natural and effective communication.
\end{abstract}

Key words: English, classroom communication, traditional education, dissonance, guidance theories 
Estudios Pedagógicos, Número Especial 40 años: 103-116, 2016

PROCESO DE ENSEÑANZA APRENDIZAJE DE LA LENGUA INGLESA EN ESCUELAS PÚBLICAS CHILENAS: PRODUCCIÓN O REPRODUCCIÓN?

\section{INTRODUCCIÓN}

Todo proceso de enseñanza aprendizaje contempla componentes humanos y culturales que deben relacionarse dialécticamente entre sí. Para lograr este objetivo, no solo es necesario que en el aula el profesor construya un proceso al servicio de las necesidades e intereses de los estudiantes, sino que, además, se requiere que los docentes de todos los subsectores, bajo el alero del Ministerio de Educación, compartan los fundamentos teóricos de un proceso de enseñanza-aprendizaje construido en post del desarrollo de la personalidad de los aprendientes. De este modo, resulta más factible contribuir a la formación integral de individuos dispuestos a transformarse en mejores ciudadanos.

No obstante, la inmersión de la sociedad chilena en el mundo globalizado neoliberal ha impuesto al proceso de enseñanza-aprendizaje de la lengua inglesa la exigencia de alcanzar la competencia comunicativa de los estudiantes en esa lengua. Al respecto, el Ministerio de Educación (MINEDUC) propone como principal objetivo de este proceso, que es igualitario para todos los aprendientes de las escuelas públicas, desarrollar las cuatro habilidades de comprensión auditiva, comprensión de lectura, producción oral y producción escrita. De igual manera, este proceso contempla desarrollar en los estudiantes su formación integral mediante la educación en valores y el contacto con las culturas extranjeras. El objetivo último es lograr que los estudiantes se comuniquen en forma fluida, y que puedan, de esta forma, responder exitosamente a los desafíos de la globalización.

Por otro lado, durante varias décadas y con el propósito de lograr los fines comunicativos que proponen las bases curriculares, un número significativo de profesores que enseñan la lengua inglesa en las escuelas públicas chilenas, ha optado por las tendencias pedagógicas tradicionalistas. Según la evidencia científica, el proceso de enseñanza y aprendizaje de la lengua inglesa en este sector se ha focalizado en lo instructivo por sobre lo educativo, limitando así a los estudiantes a la mera reproducción mecánica de los contenidos. Después de más de 40 años, sigue siendo una prioridad plantearse las interrogantes propuestas por Pilleux en el año 1976: ¿Cómo permitir que los estudiantes descubran las reglas de la lengua a través de sus errores? y ¿cómo lograr que los estudiantes internalicen las reglas para luego utilizarlas en la producción de mensajes? Desde entonces, se ha llevado a cabo un proceso destinado a la "acumulación de conocimientos y no así a la formación integral, a las transformaciones y desarrollo de la personalidad" (Ginoris, Addine y Turcaz, 2009: 164).

El objetivo de este trabajo, entonces, consiste en estudiar críticamente las disonancias que presenta el proceso de enseñanza-aprendizaje de la lengua inglesa en las escuelas públicas chilenas, con el fin de contribuir a la reconstrucción de un proceso eficaz, en el que se cumpla la hipótesis que estipula que "el conocimiento que el profesor tiene de los mecanismos naturales del aprendizaje de sus estudiantes y de su actuación en concordancia con éstos, es más importante que la aplicación ciega e irreflexiva de determinados métodos o técnicas" (Pilleux, 1976: 83). Posteriormente, este artículo plantea la urgente necesidad de determinar cooperativamente los fundamentos teóricos de la pedagogía chilena, como un paso determinante para alcanzar una educación de calidad.

\section{CARÁCTER SISTÉMICO DEL PROCESO DE ENSEÑANZA APRENDIZAJE}

El proceso de enseñanza aprendizaje está compuesto por dos interrelaciones fundamentales: los componentes humanos y los componentes culturales. Los componentes humanos lo 
constituyen el maestro, los estudiantes y el grupo; los componentes culturales abarcan los objetivos, los contenidos, los métodos, los medios, la evaluación y las formas de organización. Según González, Recarey y Addine (2004, cit. en Addine, 2004), el proceso de enseñanzaaprendizaje, como sistema, se caracteriza principalmente por la integridad de sus componentes.

Los objetivos (¿para qué enseñar? y ¿para qué aprender?) son los componentes rectores que orientan el proceso hacia el estado deseado, y poseen tres elementos: el conocimiento, la habilidad y la valoración. "[C]onstituye[n] el modelo pedagógico del encargo social, son los propósitos y aspiraciones que durante el proceso [...] se van conformando en el modo de pensar, sentir y actuar del estudiante" (Alvarez de Zaya, 1992: 58, citado en González, Recarey y Addine, 2004: 61). Los contenidos (¿qué enseñar y ¿qué aprender?) tienen relación con los conocimientos, las habilidades, los hábitos, los valores, las normas y las expresiones creativas, y permiten a los estudiantes desarrollar la capacidad de aprender a conocer, aprender a hacer, aprender a vivir juntos y aprender a ser. El método (¿cómo enseñar? y cómo aprender?) resulta ser el elemento más difícil y complejo, y para responder dialécticamente al proceso, debe ser de carácter productivo, creativo y participativo, entre otros rasgos relevantes. Los medios (¿Cómo y con qué enseñar? y ¿Cómo y con qué aprender?) se relacionan directamente con los métodos; facilitan el proceso de enseñanza-aprendizaje mediante, por ejemplo, el empleo de realia (objetos reales). La evaluación responde a la pregunta ¿en qué medida se han alcanzado los objetivos?, constituyendo un componente esencial del proceso, por cuanto determina no solo el nivel de avance de los estudiantes sino también la calidad del proceso que conduce el profesor. Por este motivo, también debe contribuir a la formación integral del estudiante y al desarrollo de su personalidad. Finalmente, las formas de organización, logran integrar todos los componentes del proceso de enseñanza aprendizaje y reflejan las relaciones que se originan entre el docente y sus aprendientes mediante la clase, la tutoría, la salida a terreno, entre otras posibilidades. De esta manera, los componentes del proceso logran relacionarse de manera sistémica y dialéctica, como lo esquematiza la siguiente figura:

Figura 1: Proceso de Enseñanza Aprendizaje (González, Recarey y Addine, 2004: 56).

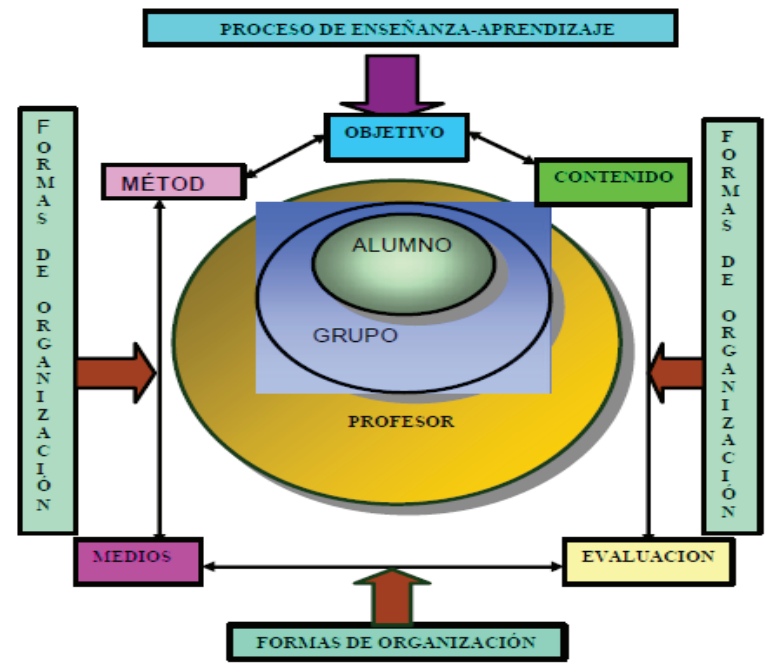


Estudios Pedagógicos, Número Especial 40 años: 103-116, 2016

PROCESO DE ENSEÑANZA APRENDIZAJE DE LA LENGUA INGLESA EN ESCUELAS PÚBLICAS CHILENAS: PRODUCCIÓN O REPRODUCCIÓN?

De acuerdo a Ginoris, Addine y Turcaz (2009), el proceso de enseñanza-aprendizaje debe responder a las problemáticas educativas que traen consigo las tendencias tradicionalistas. Se sugiere que las nuevas generaciones de estudiantes tengan la capacidad de "asimilar activa y críticamente los contenidos de la cultura y se apropien de aquellos modos de pensar, sentir y de hacer, que garanticen la orientación inteligente en contextos locales y globales complejos" (2009: 9-10). También se visualiza la educación como un proceso para preparar a los estudiantes a enfrentar los nuevos tiempos, incentivándolos a "aprender a conocer, a hacer, a convivir y a ser, en los que se centra el desarrollo de la persona" (2009: 9). Vale la pena mencionar que, según los autores, aprender a conocer significa hacer suyos "los procedimientos y estrategias cognitivas, metacognitivas y motivacionales que permitan producir el saber, resolver problemas, aprender a aprender de manera permanente, a lo largo de toda la vida y en diferentes contextos y situaciones" (González, 2009: 10). En conclusión, se sostiene que "[el] proceso de enseñanza aprendizaje en general constituye en sí mismo un proceso de formación de la personalidad del estudiante, a partir de que en el mismo se forma y desarrollo la relación entre lo cognitivo y lo afectivo (González, 2009: 12).

Según Acosta,

una didáctica desarrolladora de lenguas extranjeras concibe la enseñanza y el aprendizaje contemporáneos de excelencia como un proceso social interactivo de comunicación, formativo, holista e integrador, con una fuerte base cognitiva y esencialmente humanista, donde un grupo de estudiantes disfrutan y se responsabilizan en condiciones favorables del aprendizaje, con la solución de tareas que satisfacen sus necesidades, en un marco de socialización y cooperación que les permite auto realizarse y experimentar cambios duraderos en su actitud, actuación y pensamientos, transferibles a nuevas situaciones y producidos por la actividad práctica e intelectual en un proceso de desarrollo de competencias, creado, facilitado y dirigido por el maestro (Acosta, 2005: 6. Cit. en González, 2009: 24).

En este contexto educativo, el rol del maestro debe ser redefinido. Es él quien debe organizar el proceso de manera que cada estudiante tenga un rol protagónico y se sienta motivado y busque creativamente el conocimiento al ritmo que le corresponda (Silvestre y Zilberstein, 2003). Por otro lado, el desarrollo del estudiante, como un producto de la sociedad y de las vivencias históricas, depende de la cultura que posee el grupo con el cual se relaciona y de las relaciones sociales. Consecuentemente, los estudiantes deben trabajar con el otro para desarrollar competencias sociales tales como ayudar, tolerar y respetar a los demás (Rico, 2003: 43). Se estimula, por ende, la organización de situaciones comunicativas de aprendizaje provenientes de problemas reales y significativos para los estudiantes, con el fin de motivarlos y que amplíen su zona de desarrollo próximo (Pulido, 2005: 71). En una educación desarrolladora, se va guiando, estimulando, "ampliando continuamente los límites de la zona de desarrollo potencial y los progresivos niveles de desarrollo del sujeto" (Reinoso et al, 2012: 11). Según Vygotsky, "lo que un niño es capaz de hacer hoy con ayuda de alguien, mañana podrá hacerlo por sí solo" (1979: 134).

El profesor de la lengua extranjera "debe y tiene que concebirse como un educador que utiliza los medios de la lengua extranjera que enseña no sólo como medio de comunicación, sino también, como medio de asimilación de la cultura que dicha lengua es portadora y los valores que ella puede transmitir a los estudiantes en el proceso de su aprendizaje" (González, 2009: 15-16). Consecuentemente, no solo es necesario que el profesor posea un 
alto conocimiento de la lingüística que caracteriza la lengua que imparte, sino que también es primordial que conozca la didáctica de dicha lengua. Con el fin de que el proceso de enseñanza aprendizaje de la lengua extranjera se caracterice por su carácter educativo e instructivo, el profesor debe estar altamente preparado y contar con la experiencia necesaria que le haya permitido poner en práctica y adaptar los principios lingüísticos y didácticos. Ningún maestro que aspire a ser verdaderamente competente puede mantenerse al margen de los presupuestos teóricos que avalan la principal actividad que desarrollan: la clase (González, 2009: 11).

A pesar de que la historia de la didáctica de la lengua inglesa le haya otorgado una valor extraordinario a los métodos, el éxito de la clase, "depende del uso que se haga de los métodos, no hay métodos ni técnicas buenos ni malos" (Pilleux, 1976: 83). Independiente de éstos, el profesor debiera considerar el cómo permitir que a partir de la ejercitación mecánica, los alumnos puedan construir oraciones naturales y en contexto, y el cómo pueden llegar a comprender lo que no han escuchado antes. El profesor es quien debe velar por mantener el carácter sistémico del proceso de enseñanza aprendizaje.

\section{EL PROCESO DE ENSEÑANZA APRENDIZAJE DE LA LENGUA INGLESA EN ESCUELAS PÚBLICAS CHILENAS}

Según Yilorm y Acosta (2016), los profesores de inglés, en particular aquellos que ejercen en las escuelas públicas con estudiantes cuya situación social de desarrollo es crítica, no han podido, en general, responder significativamente a los requerimientos del Ministerio, ni a las necesidades e intereses de los aprendientes. Todo indica que estos profesores chilenos han optado por las tendencias tradicionalistas. De acuerdo a Ginoris, Addine y Turcaz (2009: 163-164), algunas de las manifestaciones de estas tendencias en el proceso educativo son: la internalización pasiva de contenidos, la reproducción mecánica, los aprendizajes memorísticos, los criterios curriculares que no suplen las necesidades de desarrollo de todos los estudiantes y/o del grupo de curso, la focalización en el proceso de enseñanza por sobre la calidad del aprendizaje, el maestro como poseedor y transmisor de conocimiento, la comunicación unidireccional, los libros como fuentes prácticamente exclusivas de contenido y los materiales educativos poco significativos. En conclusión, se observa un nulo desarrollo de los procesos metacognitivos.

Esta problemática fue planteada en el primer encuentro de investigadores chilenos en el campo de la enseñanza del inglés como lengua extranjera (Profesores de inglés, comunicación personal, 2015). También lo reconocen otros autores, los que opinan que además "no cabe duda que la mayoría de los países latinoamericanos están experimentando desafíos importantes en su sistema educacional, debido a que la pujanza de la globalización y los estándares internacionales, han impuesto exigencias significativas en las políticas nacionales" (Abrahams y Farías, 2010: III). En su análisis, Vera ("en línea") sostiene, que el proceso de enseñanza aprendizaje de la lengua inglesa, de manera general, se caracteriza en parte por ofrecer una “'enseñanza memorística', [. . .] que se destaca más bien por bajos resultados académicos, una importante falta de motivación, y además, una notoria pérdida del 'interés por aprender'”. Según Rodríguez (2004), existe, por ende, un gran número de críticas que apuntan a que el sistema educativo actual es muy descontextualizado y teoricista (Cit. en Vera, “en línea”). 
Por un largo tiempo, se ha otorgado mayor relevancia a la construcción de conocimientos como procesos personales que a los aspectos sociales y contextuales que los rodean. Como consecuencia, "[esto] no ha permitido comprender bien la conexión entre la enseñanza y el aprendizaje como una unidad dialéctica" (Ginoris, Addine y Turcaz, 2009: 162). Se lo ha considerado, más bien, un proceso destinado a la "acumulación de conocimientos y no así a la formación integral, a las transformaciones y desarrollo de la personalidad" (2009: 164). Según plantea Pilleux, "[la] aparición de una nueva corriente lingüística siempre va aparejada con un gran entusiasmo por parte de los profesores que enseñan idiomas extranjeros" (1976: 81). No obstante, en Chile, aún hay indicios de que "[la] revisión muy somera de la literatura disponible al respecto deja una sensación de desalentadora falta de progreso en cuanto a la utilidad de los métodos y técnicas que se han empleado" (Ibid.). Por lo demás, el autor plantea que existen efectivamente asuntos que los profesores de inglés en las escuelas públicas han ignorado y que deben ser considerados para lograr un proceso de enseñanza aprendizaje de la lengua inglesa eficaz: la capacidad innata y la creatividad de cada estudiante para adquirir una lengua extranjera, los errores como componentes esenciales del proceso, las transformaciones paulatinas de las estructuras bases por parte del estudiante y la capacidad de internalizar las reglas. Se observa que la gran mayoría de los profesores de inglés en las escuelas públicas prefieren las prácticas pedagógicas tradicionales, en las que todas las categorías didácticas (objetivos, contenidos, métodos, medios, organización y evaluación) se basan primordialmente en el aprendizaje de estructuras gramaticales, lo cual no favorece el aprendizaje natural de la lengua extranjera.

En relación al primer componente del proceso de enseñanza-aprendizaje de la lengua inglesa, los objetivos planteados por los profesores en las planificaciones no son coherentes con los que realmente se aplican en el aula. En los documentos escritos (MINEDUC, "en línea"), los objetivos de aprendizaje se determinan prioritariamente en base a las cuatro habilidades comunicativas, y se espera que los estudiantes sean capaces de reproducir, comprender, expresar, contrastar, describir, formular, y producir en forma oral y escrita, lo cual pone en evidencia un proceso de enseñanza aprendizaje que no se limita a la entrega de conocimientos, sino más bien, a la construcción en base a lo aprendido. No obstante, en la práctica, los estudiantes deben ser capaces de identificar vocabulario y estructuras gramaticales por medio de la traducción y explicaciones teóricas, es decir, deben alcanzar la competencia comunicativa en contextos educativos aislados y descontextualizados. Cabe mencionar que la evidencia científica ha demostrado que este objetivo no se ha podido cumplir. Los estudiantes de escuelas públicas chilenas no pueden comunicarse en la lengua inglesa.

Es también tarea del profesor definir los contenidos, en otras palabras, los conocimientos y las experiencias creadoras que deben ser adquiridos; así como también los hábitos, las habilidades y los valores que deben ser formados. Es necesario recordar que el proceso de enseñanza-aprendizaje "persigue que los alumnos se apropien de nuevos conocimientos, los fijen y apliquen a nuevas situaciones, desarrollen habilidades y hábitos y comprueben el nivel de sus conocimientos" (Labarrere y Valdivia, 2002: 37). Para poder lograr el nivel de aplicación es necesario que estos contenidos respondan a los intereses y necesidades de los estudiantes, a sus edades y a su desarrollo cognitivo y emocional.

Explicar los contenidos lingüísticos a los estudiantes, en particular a los niños, resulta complejo y aplicar ejercicios descontextualizados, y no a situaciones reales o similares a las experiencias de la vida diaria, no tiene sentido, ya que no es un contenido que corresponde 
al nivel de desarrollo de un niño. "A los estudiantes no se les enseña la lengua. Más bien éstos extraen las reglas gramaticales (y gran parte del léxico) de la lengua que se habla a su alrededor" (Fromkim, Rodman y Hyams, 2003: 390).

De acuerdo a Silvestre y Zilberstein (2003), los métodos y los procedimientos se seleccionan dependiendo de los objetivos y los contenidos. De ahí la importancia de hacer referencia a los métodos que tienden a utilizar los profesores de las escuelas públicas chilenas. Los métodos más utilizados son los de Gramática-Traducción y Audiolingüe (Ramos y Espinoza, “en línea”; Vera, 2008; Yilorm y Lizasoain, 2012), pero se agregan componentes esenciales de enfoques comunicativos, lo cual produce una contradicción significativa en las formas de organizar el proceso.

De acuerdo a Richard-Amato, el método Gramática-Traducción, también conocido como el "Método Prusiano", se destaca en Europa y América desde mediados del siglo XVI hasta mediados del siglo XX (2003:16). Este método se basa en la forma en que las lenguas latina y griega se impartían, y en la enseñanza de la lectura de textos literarios en la lengua extranjera (Ariza et al, 2002: 230), con su posterior traducción a través de un listado de palabras y reconocimiento de las estructuras gramaticales (Brown, 1994). Originalmente, el objetivo principal de este método era lograr que los estudiantes lean y escriban en la lengua extranjera tras el aprendizaje de las reglas y sus respectivas aplicaciones (Richard-Amato, 2003: 16). En la actualidad, los profesores de inglés en las escuelas públicas tienden a desarrollar las cuatro habilidades de manera simultánea en sus estudiantes, sin otorgarle un rol prioritario a la habilidad comprensión auditiva.

El método Audiolingüe fue introducido como un componente de un método de la armada, debido a que la afiliación de los Estados Unidos a la Segunda Guerra Mundial impactó significativamente el proceso de enseñanza aprendizaje de lenguas en América. La armada necesitaba personal que pudiera comunicarse fluidamente en diferentes lenguas y así fue como se le solicitó a las universidades americanas que desarrollaran un programa de enseñanza-aprendizaje de la lengua inglesa. En ese entonces, Bloomfield, junto a otros lingüistas, ya habían desarrollado programas de capacitación, que consistían principalmente en repetir oraciones emitidas por el profesor. Estos acontecimientos en los años 50, dieron origen al método Audiolingüe (Hamer, 1991; Richards, 2001; Richards y Rodgers, 2008).

Para Charles Fries, la gramática, o la estructura, definían el punto de partida en el aprendizaje de la lengua. "La lengua era enseñada con especial énfasis en la pronunciación y por medio de una ejercitación oral extensiva de patrones con oraciones básicas" (Richards y Rodgers, 2008: 52). La aplicación de este método en la actualidad consiste en la repetición de palabras, frases y/u oraciones. Al aplicar este método, la comprensión auditiva y las habilidades orales son objeto de atención. No obstante, las palabras u oraciones que se deben repetir generalmente no tienen relación con eventos o narraciones relacionados con la vida actual.

De esta manera, Richard-Amato afirma que "el método Audiolingüe encuentra su origen en la teoría del conductismo y se adhirió al pensamiento de que la lengua se adquiere a través de la formación de hábitos y de la asociación estímulo-respuesta" (2003: 17). En la misma línea de investigación, se sostiene que el lenguaje es considerado un proceso de formación de hábitos que refleja la teoría de la psicología conductual (Ariza et al, 2002). "[Los estudiantes] requieren ejercitación y más ejercitación y solo una cantidad suficiente de vocabulario para hacer posible la ejercitación" (Richards y Rodgers, 2008: 52). Se concluye que la psicología conductista niega el rol de la conciencia y conduce a 
Estudios Pedagógicos, Número Especial 40 años: 103-116, 2016

PROCESO DE ENSENAANZA APRENDIZAJE DE LA LENGUA INGLESA EN ESCUELAS PÚBLICAS CHILENAS:

PRODUCCIÓN O REPRODUCCIÓN?

un proceso de enseñanza-aprendizaje mecanicista que no formula objetivos cognoscitivos (Antich, 1988).

Las principales críticas consisten en que, mediante este método, se explican solo los aspectos más superficiales de la lengua extranjera, desconociendo las estructuras más profundas. No existe un cuestionamiento acerca de cómo un estudiante puede captar las reglas y luego aprenderlas. En conclusión, el estudiante solo repite mecánicamente. "Es indudable que la capacidad creativa del hablante no puede ser producto de una repetición mecánica de frases y oraciones" (Pilleux, 1976: 82).

A nivel internacional, estos métodos son considerados obsoletos. No obstante, para el profesor de inglés en Chile, éstos representan una opción cómoda y eficaz de impartir sus conocimientos. El profesor es una autoridad que se enfrenta a un grupo curso siempre organizado de la misma manera: en varias filas, una tras la otra. Ante este hecho, los estudiantes supuestamente demuestran más respeto y responden en forma más efectiva. No se requiere preparar diversas actividades, ni poner en práctica otras formas de organización del proceso. Completar los ejercicios del libro y/o una guía de trabajo extensa y a la vez compleja, ya que abarca estructuras gramaticales, puede mantener a los estudiantes más atentos y concentrados por más de una clase de 90 minutos. De esta misma forma, la repetición coral de las palabras y oraciones es una manera efectiva de llamar la atención de los estudiantes que saben cómo responder a las estrategias tradicionales desde la edad preescolar. Como no se utiliza la lengua extranjera, no se requiere de parte del profesor un esfuerzo para hacerse comprender; más bien mantienen al profesor estático en su desarrollo profesional. Bajo este contexto, Richard-Amato sostiene que: "no [habría] intensión de lograr comunicación oral y/o escrita en la lengua extranjera” (2003: 17).

El proceso de enseñanza-aprendizaje de la lengua extranjera debe, por su naturaleza, promover relaciones afectivas y significativas en el aula. Éstas permiten que los estudiantes se relajen, se sientan cómodos y alegres. De igual manera, este estado emocional motiva a los estudiantes a participar más activamente (Hastings, comunicación personal, 2007). No obstante, la figura del profesor como autoridad que dirige todas las actividades difícilmente puede favorecer las relaciones humanas en el aula. Los estudiantes más bien cumplen un rol reactivo al responder a los estímulos, y por lo tanto, no tienen incidencia en el proceso de enseñanza-aprendizaje que, por naturaleza, es bidireccional.

Es un hecho que los medios responden a las características de los objetivos, contenidos, métodos y procedimientos. No sorprende que los materiales didácticos utilizados en las escuelas públicas chilenas sean utilizados tradicionalmente. Los textos de estudio, las guías de aprendizaje, el pizarrón, el equipo de música, el computador y el data show predominan en el aula y son utilizados de manera descontextualizada. Según los datos empíricos, la mayor deficiencia de esta realidad es que los profesores favorecen el desarrollo del estilo visual de aprendizaje y se descarten arbitrariamente los demás, problemática que podría resolverse mediante el empleo de los materiales didácticos multisensoriales. Es sabido que mientras más sentidos estén incorporados en el proceso de enseñanza-aprendizaje, más se beneficia la apropiación de los saberes (Logsdon, comunicación personal, 2013).

La forma de organización es determinante para que el maestro imparta clases de calidad. Éstas deben proveer espacios que permitan que los miembros del aula se relacionen entre sí y trabajen colectivamente para alcanzar los objetivos de aprendizaje. Las características del proceso de enseñanza aprendizaje de la lengua inglesa en el contexto que se estudia, limitan las formas de organización al desarrollo principalmente de la clase 
expositiva, en la que se desarrolla un mínimo de actividades de larga extensión y que tratan los conocimientos, hábitos y las habilidades de manera uniforme. Esta única alternativa excluye a los estudiantes que han desarrollado otras formas de inteligencias que no sean las más lógicas, y no dan lugar al trabajo en equipo, sino más bien al relleno de ejercicios mecánicos que se presentan en una guía de trabajo y que se llevan a cabo únicamente en el aula. Estas estrategias, sin duda, fortalecen el individualismo, dificultando el desarrollo de la personalidad.

Siguiendo esta forma de organización, es posible afirmar que las evaluaciones tienen un rol importante, ya que determinan el éxito académico y social de los estudiantes. Son presentadas de manera aislada, como actividades desconectadas del proceso de enseñanzaaprendizaje. En primer lugar, al no realizar la evaluación diagnóstica o al llevarla a cabo como una actividad administrativa, no es posible para el profesor prestar una atención diferenciada a quien lo necesite, y así alcanzar un nivel mínimo común de partida. La ausencia de actividades específicas que fortalezcan la convivencia escolar le otorga a la evaluación en muchas ocasiones un carácter punitivo, lo cual quebranta la interrelación entre las categorías del proceso de enseñanza-aprendizaje. En este proceso, la evaluación en el aula responde a los objetivos de aprendizaje basados en la gramática explícita, lo que deja en evidencia su alto grado de dificultad.

Los deficientes resultados académicos de los estudiantes chilenos que asisten a las escuelas públicas parecen ser productos legítimos de un sistema educacional arcaico, propio a su vez del modelo neoliberal. En general, no se favorece el desarrollo cognitivo, social y afectivo de los estudiantes, limitándolos más bien a reproducir conocimientos y no así a crearlos. Cabe mencionar que una concepción tradicionalista del proceso de enseñanza aprendizaje de la lengua no responde a las necesidades del mundo actual.

\section{CARACTERIZACIÓN DEL PLAN DE ESTUDIO PARA ESTUDIANTES DE SEXTO BÁSICO DE ESCUELAS PÚBLICAS CHILENAS}

Para la realización de este epígrafe, se tuvo en cuenta el análisis de documentos que emite el Ministerio de Educación en Chile y que constituyen regulaciones que han de ser consideradas por la escuela. Este documento es el Programa de estudio para el idioma extranjero inglés de sexto año básico. El desarrollo integral de los estudiantes chilenos contempla el proceso de enseñanza-aprendizaje del inglés como lengua extranjera. El propósito del Ministerio es que los estudiantes chilenos desarrollen habilidades comunicativas en forma oral y escrita y, de esta forma, puedan participar activamente en los procesos socioeconómicos y políticos de la globalización. Sin embargo, no hay una adecuada disposición que oriente el tratamiento didáctico requerido para desarrollar esas habilidades comunicativas, que en el programa de estudios, aparecen en forma dispersa y solo se limita a considerar que el proceso se ha de organizar de modo que se logre el desarrollo de las cuatro habilidades en forma equitativa y simultánea.

El objetivo general del programa es que los estudiantes de sexto básico desarrollen la competencia comunicativa, es decir, su capacidad de comunicarse en inglés de manera significativa y contextualizada a partir del desarrollo de las cuatro habilidades (escuchar y leer comprensivamente y producir en forma oral y escrita). A su vez, los objetivos de aprendizaje específicos de cada clase consideran todos los componentes de la lengua 
Estudios Pedagógicos, Número Especial 40 años: 103-116, 2016

PROCESO DE ENSEÑANZA APRENDIZAJE DE LA LENGUA INGLESA EN ESCUELAS PÚBLICAS CHILENAS:

PRODUCCIÓN O REPRODUCCIÓN?

(vocabulario, temáticas, estructuras gramaticales, estrategias y funciones, entre otras) y se proponen que los estudiantes utilicen el idioma para interactuar.

Estas metas resultan ser muy difíciles de alcanzar para la realidad chilena, especialmente en los contextos con una situación social de desarrollo en los cuales los estudiantes comparten vivencias dramáticas que impactan su identidad, integridad y dignidad. A modo de ejemplo, en una clase cuyo objetivo es desarrollar la comprensión auditiva, se sugiere empezar con preguntas orales relacionadas con el tema a las cuales los estudiantes deben responder en forma oral. Luego, se propone introducir un texto en relación al cual los estudiantes podrán contestar preguntas en forma escrita. Sin duda que en este ejemplo se percibe la interrelación de las cuatro habilidades en forma permanente. No obstante, previo a determinar los objetivos, es menester tener en consideración el nivel mínimo común de partida. ¿Cómo contestar preguntas si no se comprenden los textos orales porque el conocimiento del mundo es limitado, porque no se posee el léxico suficiente, porque no hay capacidad ni interés para predecir y adivinar, porque los estudiantes no poseen estrategias que les permitan identificar las ideas generales de un texto y porque no hay desarrollo de las habilidades que permiten inferir estados de ánimos.

Los objetivos transversales apuntan al desarrollo de actitudes tales como la curiosidad, el interés, el estilo de trabajo y las estrategias, la actitud positiva frente a sí mismo y el respeto ante los demás. Estos objetivos son mucho más complejos de alcanzar en contextos con desventaja social, por lo cual se requiere de un trabajo profundo, progresivo y a largo plazo que esté en funcionamiento permanente en cada objetivo de aprendizaje. No se observa en el programa de estudio una orientación didáctica que permita el diseño e implementación de un plan de convivencia escolar que fortalezca estos objetivos transversales.

Los contenidos de sexto básico son presentados en cuatro unidades de trabajo, con una estimación de tiempo de 28 horas pedagógicas por unidad; estas son: Food and Health, Around the Town, The Natural World, y Let's Travel. En relación a los contenidos lexicológicos, se estima que estos debieran ser analizados con el fin de determinar si temáticas tan complejas como la unidad de comida debiera ser incorporada en un contexto donde se presenta como una necesidad inmediata. De la misma manera, la autora recomienda tener especial cuidado con la forma en que se presenta la unidad Let's Travel, considerando que viajar y vacacionar no son derechos sociales en Chile, sino más bien privilegios de quienes poseen los recursos.

En cuanto a la cantidad de palabras que se recomienda introducir, cabe señalar que en el reconocido programa de enseñanza aprendizaje de lenguas extranjeras, Foreign Languages in Elementary School (FLES) en el Condado de County, Virginia, Estados Unidos, en el cual se desarrolla en primer lugar la habilidad comprensión auditiva, se trabaja con un máximo de ocho a diez palabras por unidad. No obstante, los planes y programas de este mismo nivel en Chile, requieren que los estudiantes adquieran alrededor de cuarenta palabras por unidad en una misma cantidad de tiempo. Es sabido que los niños no están capacitados biológicamente para retener y utilizar en contexto esta cantidad de palabras en el tiempo que determina el programa. Muy por el contrario, se estima que las palabras no deben ser muchas y que deben repasarse una y otra vez, y de diferentes maneras.

En términos gramaticales, el programa de estudio presenta deficiencias en cuanto al orden natural en que se aprende una lengua. Por ejemplo, en el caso del nivel sexto año básico, el primer contenido es el presente simple en sus tres tipos de oraciones (afirmación, negativo y pregunta), no siendo éste el primer tiempo verbal que se adquiere en forma 
natural (Ariza et al., 2003). Por otro lado, el presente simple en su forma afirmativa requiere aproximadamente entre 6 y 8 años para ser adquirido en su totalidad (Krashen, 1998). También se sugieren en el programa que al finalizar el año escolar, los estudiantes se expresen en el presente simple, futuro y pasado con todas sus características, utilicen los verbos modales can y cay, el uso del imperativo, adverbios de frecuencia y tiempo, verbos más gerundio, conectores because, and, but y or, secuenciadores comofirst, then, later, entre otros, there is/there are, preposiciones de lugar y adjetivos descriptivos y comparativos. Desde el punto de vista lingüístico y metodológico, resulta imposible apropiarse de todos estos contenidos en un año escolar.

Se promueven diversos métodos y enfoques para el proceso de enseñanza aprendizaje de la lengua inglesa. No obstante, el enfoque comunicativo es el que se recomienda en todo momento. El enfoque natural, el enfoque basado en la tarea, el enfoque basado en contenidos, y el enfoque de aprendizaje cooperativo del lenguaje cumplen la función de complementar el liderazgo del enfoque comunicativo. Por ende, en todo momento se aprecia el desarrollo de las cuatro habilidades en forma integrada desde el primer día. No se aprecia una inclinación hacia un proceso de enseñanza-aprendizaje natural en el que los niños aprenden por medio de lo que más les interesa: jugar. Tampoco se observa una preocupación particular por el período de silencio en el que los estudiantes solo escuchan y observan. No se hace referencia a los métodos y procedimientos para un desarrollo óptimo de la convivencia escolar.

Si bien se menciona el uso de distintos materiales, como el diario y los programas de televisión, y el uso del libro como un material didáctico de apoyo al trabajo del docente, el texto de estudio es finalmente el material más significativo para el docente, debido a que el Ministerio de Educación lo distribuye en las escuelas públicas. Existe una gran controversia con respecto a su uso y a los efectos que cumple en el aula. Es un material didáctico que, si bien proviene del Ministerio, no se ajusta a los enfoques comunicativos que se promueven. El texto de estudio no desarrolla las habilidades comunicativas de los estudiantes en forma contextualizada. Se observan ejercicios de gramática explícita en forma reiterada, las temáticas no han sido plenamente adaptadas a los intereses y necesidades de los estudiantes chilenos; en conclusión, no se respetan las etapas del proceso de aprendizaje de los estudiantes. Muy por el contrario, se requiere el dominio de contenidos, que normalmente son aprendidos progresivamente, en forma rápida y descontextualizada. Adicionalmente, no se percibe referencia alguna al diseño y creación de material didáctico multisensorial.

Se promueven las actividades en pareja, los juegos de roles, la solución de problemas, y la participación en proyectos grupales, entre otros. No se observa, sin embargo, la intención de promover el uso significativo de actividades comunicativas lúdicas e interactivas en el aula y fuera de ella. Tampoco hay referencia a la formas de organización para la convivencia escolar.

Se sugiere una serie de actividades para la evaluación de los contenidos con el fin de considerar los distintos estilos de aprendizajes, tales como las presentaciones, los informes orales y escritos, los portafolios, las pruebas orales y escritas, y los controles, entre otros. Sin embargo, no se considera la evaluación como un proceso natural de aprendizaje en la que el estudiante participa activamente y en el que disminuye notoriamente la cantidad de evaluaciones formales. Sigue habiendo un predominio importante de las formas tradicionales de evaluación. Como primera sugerencia para el diseño de la evaluación, el programa sugiere partir considerando los objetivos de aprendizaje y los indicadores de 
evaluación que se han sugerido en el programa, lo cual refleja una cierta incoherencia con respecto a considerar el documento como opcional y a la libertad de cada docente para adaptar el programa de estudio a su propia realidad.

La principal problemática relacionada con este plan de estudios es que la gran mayoría de los profesores de inglés solo considera de este programa de estudio los objetivos de aprendizaje y el texto de estudio como material didáctico principal que, además, provee los contenidos de la asignatura. Por las razones mencionadas anteriormente, no se consideran las orientaciones didácticas que se sugieren, tales como los métodos, los procedimientos, la forma de organización y la evaluación, lo cual de alguna manera inhabilita el programa y produce una desestabilización en el proceso de enseñanza-aprendizaje de la lengua inglesa.

Todo indica que, desde el punto de vista administrativo, seguir el programa al pie de la letra, tal cual es presentado por el Ministerio de Educación, es un requerimiento. Cabe recordar que ciertos establecimientos educacionales públicos ya se encuentran trabajando con el sistema de planificación en línea en base a objetivos ya determinados que provienen de los programas de estudio.

Es evidente que el Ministerio de Educación no contempló que, bajo el alero de un Apartheid educativo ocasionado por el modelo socio-económico neoliberal imperante, los docentes de inglés que imparten clases en las escuelas públicas no se caracterizan por adaptar el programa a sus realidades, porque el agobio laboral no permite que se dedique el tiempo y la energía necesarios. El plan de estudios tampoco ha considerado los estudios que se han llevado a cabo para determinar el estado del arte del proceso de enseñanza aprendizaje de la lengua inglesa en Chile, el cual ha indicado, desde el año 2004, que el porcentaje de estudiantes chilenos que asisten a escuelas públicas y que comprenden oraciones simples y básicas es mínimo.

\section{DISCUSIÓN}

Con el fin de responder satisfactoriamente a las demandas de la globalización, el proceso de enseñanza-aprendizaje de la lengua inglesa en Chile tiene como objetivo desarrollar la competencia comunicativa de los estudiantes de las escuelas públicas, para lo cual se orienta la implementación del enfoque comunicativo y el desarrollo integral de las cuatro habilidades comunicativas. No obstante, mediante el análisis de los programas de estudio y documentos normativos del Ministerio de la Educación en Chile, se ha caracterizado una realidad escolar que refleja que el proceso de enseñanza aprendizaje de la lengua inglesa en las escuelas públicas chilenas se ha focalizado en la instrucción mediante la implementación de métodos tradicionales. Consecuentemente, un número significativo de estudiantes no puede comunicarse en la lengua extranjera.

Tal cual lo expresara la autora en el III Congreso de Educación Región de Los Ríos, la evidencia científica demuestra que se observan contradicciones reveladoras en la integración indisoluble que debe existir entre los componentes humanos y culturales del proceso de enseñanza-aprendizaje de la lengua inglesa en Chile, el cual no se ha conducido sistémicamente, y por ende, no se ha permitido que se logren los estados deseados. Estas contradicciones no solo encuentran su origen en el sistema educativo neoliberal, sino que también en la ausencia de un trabajo cooperativo entre los docentes de la lengua inglesa y el Ministerio de Educación, con el fin de establecer los fundamentos teóricos de la clase 
de la lengua inglesa, requisito fundamental para empezar la construcción de un proceso de enseñanza aprendizaje de calidad (Yilorm, 2016).

Al igual que el Dr. Pilleux (1976), la autora plantea su preocupación por la crisis educacional que ha mantenido a los docentes de la lengua inglesa focalizados en la defensa y aplicación ciega de los métodos tradicionales, lo cual no ha permitido que estos conozcan los mecanismos del proceso de enseñanza-aprendizaje de la lengua inglesa. Tal como lo plantea el autor, estamos ante la urgente necesidad de que el docente de la lengua inglesa comprenda que sus actitudes con los aprendientes son más relevantes que los métodos que se utilizan en el aula. De esto depende que el estudiante mantenga o pierda el interés necesario ante el proceso de enseñanza-aprendizaje. No obstante, existe en este trabajo una reflexión más profunda que no se limita a las decisiones y desempeño de los docentes en el aula, sino que se extiende a un proceso que se ha construido acorde al modelo neoliberal actual y que no está al servicio de los componentes humanos: los profesores y sus estudiantes.

\section{REFERENCIAS BIBLIOGRÁFICAS}

Addine, F. (2004). Didáctica: Teoría y práctica. La Habana: Editorial Pueblo y Nuevo.

Antich, R. (1988). Metodología de la enseñanza de las lenguas extranjeras. La Habana: Editorial Pueblo y Educación.

Fromkin, V., Rodman, R. y Hyams, N. (2003). An introduction to language. ( $7^{\text {th }}$ Ed.) Boston, Massachusets: Thomson Wodsworth.

Ariza, E., Morales-Jones, C., Yahya, N. y Zainuddin, H. (2002). Why TESOL? Theories and issues in teaching English as a second language with a K-12 Focus, 2a ed. Boca Raton, Florida: Kendall Hunt Pub Co.

González, R. (2009). La Clase de lengua extranjera. Teoría y práctica. La Habana: Editorial Pueblo y Educación.

Ginoris, O., Addine, F. y Turcaz, J. (2009). El proceso de enseñanza-aprendizaje desarrollador. Componentes del proceso de enseñanza-aprendizaje: Objetivo, contenidos y métodos de enseñanza-aprendizaje, en Fundamentos didácticos de la educación superior cubana. Selección de lecturas, Oscar Ginoris. La Habana: Editorial Félix Varela.

Harmer, G. (1991). The Practice of English language teaching. UK: Longman.

Labarrere, G. y Valdivia, G. (2002). Pedagogía. La Habana: Editorial Pueblo y Educación.

Ministerio de Educación (2015). Introducción a esta asignatura en los programas de estudios. Recuperado de www.mineduc.cl

Pileux, M. (1976). La enseñanza de un idioma extranjero: ¿Teoría o Técnica? Estudios Pedagógicos, vol. 1 (1), 81-88.

Pulido, A. (2004). Hacia un concepto de competencia comunicativa integral: un novedoso acercamiento a sus dimensiones / Arturo Pulido Díaz, Vilma Ma. Pérez Viñas. Pinar del Río: Instituto Superior Pedagógico "Rafael María de Mendive". Recuperado de http://www.intranet/ CDIP/Revista\%20Mendive /Index.htm

Ramos, L. y Espinoza, M. (2008). El Profesor de inglés y su labor pedagógica en el mundo globalizado. Recuperado de http://www.sonaples.cl

Reinoso, C., Ballester, S., González, C., Torres, Y., y Ribot, E. (2012). El aprendizaje desarrollador. Teoría y práctica en la formación de educadores. La Habana: Educación Cubana. Ministerio de Educación.

Richard-Amato, P. (2003). Making it happen. From interactive to participatory language teaching. Theory and Practice. White Plains, NY: Pearson Education. 
Estudios Pedagógicos, Número Especial 40 años: 103-116, 2016

PROCESO DE ENSEÑANZA APRENDIZAJE DE LA LENGUA INGLESA EN ESCUELAS PÚBLICAS CHILENAS:

PRODUCCIÓN O REPRODUCCIÓN?

Richards, J., y Rodgers, T. (2001). Approaches and methods in language teaching. 2a.ed. United States of America: Cambridge University Press.

Richards, J. (2001). Communicative language teaching today. Cambridge: Cambridge University Press.

Rico, P. (2003). La Zona de Desarrollo Próximo. Procedimientos y tareas de aprendizaje. La Habana: Editorial Pueblo y Educación.

Vera, F. (2008). Estado del arte de la profesión de profesor de inglés: ¿Qué ocurre en Chile? Recuperado de http://www.utemvirtual.cl/nodoeducativo/wp-content/uploads/2009/03/art_ fvera.pdf

Vygotsky, L. (1979). El desarrollo de los procesos psicológicos superiores. Barcelona: Editorial Crítica.

Zilberstein, J. et al. (2003). Preparación pedagógica integral para profesores universitarios. LaHabana: Instituto Superior Politécnico José Antonio Echeverría, Cuba.

Yilorm, Y. y Lizasoain, A. (2012). Evaluación de la puesta en marcha del enfoque metodológico por competencias comunicativas, Revista Literatura y Lingüística, 25, Universidad Católica Silva Henríquez.

Yilorm, Y. (2016). Neoliberalismo y proceso de enseñanza aprendizaje de la lengua inglesa en Chile: Una mirada dialéctica del estado del arte en sectores vulnerables. Revista CEPES (en prensa).

Yilorm, Y. (2016). La relación dialéctica entre los componentes del proceso de enseñanza aprendizaje: Requisito primordial para una educación de calidad en Chile. Acta del III Congreso de Educación Región de los Ríos. Valdivia: Universidad San Sebastián. 